\title{
The Effect of Stretching on Transmural Shear Wave Anisotropy in Cardiac Shear Wave Elastography
}

\author{
Annette Caenen, Abdullah Thabit, Darya \\ Shcherbakova, Abigail Swillens, Patrick Segers \\ IBiTech-bioMMeda \\ Ghent University \\ Ghent, Belgium \\ E-mail: annette.caenen@gmail.com
}

\author{
Mathieu Pernot \\ Langevin Institute \\ Ecole Supérieure de Physique et de Chimie Industrielles \\ Paris, France
}

\author{
Luc Mertens \\ Hospital of Sick Children \\ University of Toronto \\ Toronto, Canada
}

\begin{abstract}
Shear Wave Elastography (SWE) is a potential tool for non-invasively assessing myocardial stiffness to support diagnosis and treatment choice in patients with cardiac disorders. Previous studies demonstrated a 3D anisotropic shear wave propagation in cardiac SWE due to the intrinsic myocardial fiber architecture. The aim of this work is to further investigate the performance of cardiac SWE by studying the effect of uniaxial stretching on anisotropic shear wave propagation and characterization. Results showed a clear increase in group and dominant phase speed during stretching, especially along the direction of the fiber. Additionally, the maximal group and dominant phase speed value spatially shifted while stretching, indicating an alignment of the fibers to the stretching direction. Complementary numerical modeling could further explore these interactions between myocardial fiber architecture and cardiac loading during SWE.
\end{abstract}

Keywords-cardiac shear wave elastography; anisotropy; stretching; group speed; phase speed

\section{INTRODUCTION}

The microstructural organization of the myocardium and its material properties play an essential role in the heart's physiology and pathophysiology. A non-invasive ultrasonic technique, i.e. Shear Wave Elastography (SWE), may allow the assessment of these intrinsic characteristics and therefore contribute to cardiac function evaluation in healthy and diseased conditions. Initial feasibility studies have shown the potential of SWE in (i) generating and tracking shear waves transthoracically in the myocardium [1], but also in (ii) representing the structural and material characteristics of the myocardium by analysis of shear wave characteristics $[2,3]$. In this work, we focus on the isolated effect of the myocardial fiber architecture on shear wave characterization, complementing previous work where we studied the isolated effect of left ventricular geometry [4].

This work was supported by the Flemish government agency for Innovation and Entrepreneurship (VLAIO) and Research Foundation Flanders (FWO)
The myocardium is composed of sequential layers of parallel myocytes (predominant fiber type) and matrix proteins such as collagen [5]. As it is known from physics that shear waves propagate faster along than across fiber orientation, the SWE-excited shear wave in the myocardium is anticipated to be anisotropic in 3D. This feature has been employed to extract fiber orientation in various anisotropic tissues, with validation in a.o. ex vivo pig hearts [6]. The effect of this spatial 3D anisotropy in shear wave propagation on group shear wave speed has also been studied in a freshly explanted sheep heart [2] and in in vivo ovine hearts at mid-systole [6]. Cardiac dynamics also affect shear wave propagation and characterization, as shown in [3] for Langendorff perfused rat hearts (studied at one axial depth across tissue thickness).

The aim of this work is to study the combined effect of the 3D myocardial fiber anisotropy and mechanical loading on shear wave propagation and characterization, which has not been studied yet. Furthermore, we wish to extend the shear wave analysis from the time domain to the time and frequency domain (group and phase speed analysis, respectively). The 2D group shear wave speed surfaces across tissue thickness are used to derive fiber orientations and the results were compared to those obtained from image analyses. The study is performed on ex vivo pig myocardial slabs of the left ventricle, such that complicating factors such as ventricular geometry and surrounding tissue, residual strains and stresses do not need to be accounted for.

\section{MATERIALS AND METHODS}

\section{A. Myocardial tissue}

Pig hearts $(n=4)$, obtained from a slaughterhouse, were dissected to harvest rectangular slabs of left ventricular tissue. Therefore, the atria and right ventricle were removed 
and the left ventricle was incised along the interventricular septum until the apex, as shown in the left panel of Fig. 1.

\section{B. SWE-experiments}

1) Set-up: The myocardial slabs were submerged in a warm water bath (temperature range of $36-38{ }^{\circ} \mathrm{C}$ ), and uniaxially stretched at $5 \%, 10 \%$ and $15 \%$ with respect to the zero-stretch length along the heart's circumferential direction between the clamps, as shown in the middle panel of Fig. 1. The L15-4 probe (pitch of $0.2 \mathrm{~mm}$ and 256 elements) of the Aixplorer system (SuperSonic Imagine, Aix-en-Provence, France) was placed in the center of the stretched myocardial sample to image the left ventricular free wall. Shear waves were generated by an $8 \mathrm{MHz}$ sinusoidal pulse of $250 \mu \mathrm{s}$, an F-number of 1.5 and an excitation voltage of $50 \mathrm{~V}$ while rotating the probe from $0^{\circ}$ to $180^{\circ}$ in steps of $10^{\circ}$ (total of 19 angles). A probe orientation of $0^{\circ}$ or $180^{\circ}$ corresponded to the circumferential direction of the heart as shown in Fig. 1. The shear wave propagation was imaged using 128 probe elements with a pulse repetition frequency of $6.3 \mathrm{kHz}$.

2) Data analysis:

a) Pre-processing: Axial velocities were obtained after applying an autocorrelation algorithm [7] to the Inphase Quadrature (IQ)-data. These velocities were averaged across depth every $0.6 \mathrm{~mm}$ and up-sampled in the slow time domain by a factor 10 .

b) Group velocity analysis: Shear wave propagation characteristics were studied at 30 axial paths across the myocardial slab covering $17 \mathrm{~mm}$ of tissue thickness. Group Shear Wave Speed (SWS) was calculated by the Time To Peak (TTP) algorithm [8], a time-of-flight method fitting a linear regression curve to the shear wave's tracked position, i.e. maximal tissue velocities, in function of time. For fitting, the robustfit function in MATLAB R2014a (The MathWorks Inc., Natick, MA, USA) was used. Only regression curves with an R-squared value higher than 0.9 were considered for shear wave speed calculation. Left and right SWS were averaged if both fits fulfilled this criterium.
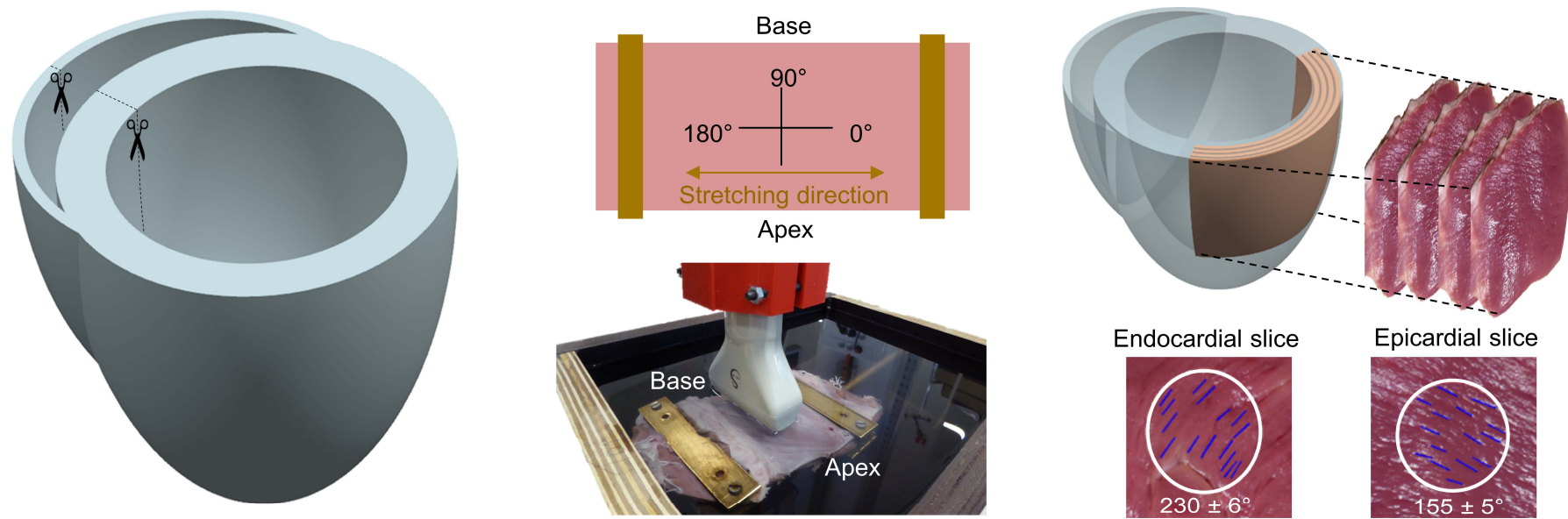

Fig. 1. Left panel - Indication of the location of tissue excision. Middle panel - Actual and schematic representation of the experimental set-up. Right c) Fiber orientation analysis: Approaching the myocardium as a composite of transversely-isotropic layers [6], fiber orientation for each layer can be derived based on the direction of fastest shear wave travel. Translating this to our experimental setting, the fiber orientation can be estimated by searching for the probe orientation yielding the highest shear wave speed for a certain shear wave path. Hence, for each sample at a given stretch, group speed analysis (see section II.B.2.b) yielded a 30x19 SWS-matrix for the considered 30 shear wave paths and 19 probe angles. As the group speed surface in a transversely isotropic material is ellipse-shaped in the plane of symmetry [9], the fiber orientation corresponds with the tilted major axis of a fitted ellipse. This ellipse fitting is performed in MATLAB in a least squares manner [10]. Mean Squared Error (MSE) was reported as a measure of the goodness of fit.

d) Phase velocity analysis: The dispersive shear wave characteristics are studied in the frequency $f$ and wave number $k$ domain by taking the 2D Fast Fourier Transform (FFT) of the axial velocities of left/right shear wave for 5 shear wave paths across tissue thickness [11]. An amplitude mask of $10 \%$ was applied to reduce the noise in the $(f, k)$ space. Excited modes were identified by tracking the wavenumbers with high Fourier energies for each frequency. The main excited mode is found by selecting the mode with the highest frequency energy content. Phase velocities $c_{\varphi}$ were calculated as $c_{\varphi}=(2 \pi f) / k$.

e) Statistical analysis: We further analyzed group and phase velocity values by performing a General Linear Model (GLM) univariate analysis in the statistical software SPSS version 24 (IBM Corp., Armonk, NY, USA). This model included the fixed factors heart (4 different samples), depth (30 paths), angle $\left(0-180^{\circ}\right)$ and stretch $(5-15 \%)$, but also the interacton terms angle*stretch and angle*depth as fiber angle varies with depth and stretch. A factor was statstically significant when it entered the model with a Pvalue $<0.001$. Estimated marginal means were calculated for the 3 stretch ratios and the 19 investigated probe angles.

\footnotetext{
panel - Extraction of myocardial fiber orientation based on image analysis.
} 

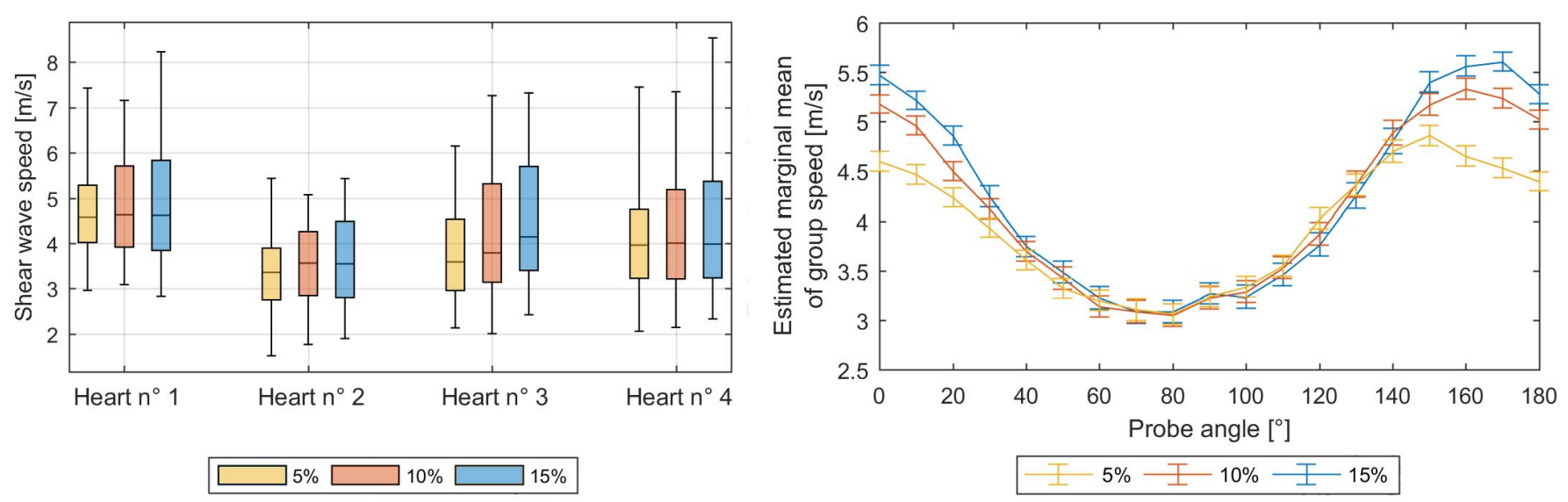

Fig. 2. Left panel - Effect of stretching on group speed values for all acquisitions with probe rotation from $0-180^{\circ}$, demonstrated in a boxplot (box represents the 3 quartiles, whiskers indicate minimum/maximum). Right panel-Combined effect of stretching and probe orientation on estimated marginal mean of group speed values for all hearts (errorbars indicate $95 \%$ confidence interval).

\section{3) Image analysis}

The myocardial fiber orientations can be studied optically due to the laminar structure of the myocardium [12]. Therefore, the ventricular slabs were cut into 4 slices of 5 $\mathrm{mm}$ thick by using an electrical meat slicer. A picture was taken of the upper and lower side of each slice (right panel of Fig. 1). On these photographs, the center of the probe was located and fibers $(n=15)$ were tracked in a circular region with radius of $12.8 \mathrm{~mm}$ (representing 128 probe elements) around this center point. This analysis was repeated for each photo, except for epi- and endocardium, leading to an average fiber angle (and standard deviation) at three different points across tissue thickness.

\section{RESULTS}

\section{A. Group speed analysis}

All group velocity data are pooled in boxplots in the left panel of Fig. 2. The $25^{\text {th }}$ and $50^{\text {th }}$ percentile do not show a clear trend with respect to stretch ratio, whereas the $75^{\text {th }}$ percentile increases with stretch for all hearts. Note the SWS variability in between hearts. Median value for hearts 3 and 4 is around $4 \mathrm{~m} / \mathrm{s}$ for all stretch ratios, while values are higher for heart $1(4.66-4.77 \mathrm{~m} / \mathrm{s})$ and lower for heart 2 (3.33-3.59 $\mathrm{m} / \mathrm{s})$. The GLM univariate analysis revealed that all defined factors entered the model: heart, angle, stretch, depth, angle*stretch and depth*angle, mentioned in order of

importance. The GLM also yields the marginal mean of the group speed for a given average sample and depth. The right panel of Fig. 2 clearly shows that group speed only increased with stretch for probe orientations $0-30^{\circ}$ and $120-180^{\circ}$ (i.e. along the fiber direction). Additionally, the probe orientation giving the maximum of the estimated marginal mean group speed value varied depending on the applied stretch: $150^{\circ}$ for $5 \%, 160^{\circ}$ for $10 \%$ and $170^{\circ}$ for $15 \%$.

The spatial anisotropy in group speed becomes apparent in Fig. 3, displaying group shear wave speed as a function of probe angle and tissue thickness (data shown for heart sample 3). This figure confirms that group speed increases when stretch increases and visualizes the variation of the maximal SWS with probe orientation across tissue thickness.

\section{B. Fiber orientation analysis}

The SWE-derived fiber orientation (colored symbols in Fig. 4) varied across tissue thickness in a range from $132.7^{\circ}$ $166.3^{\circ}$ (heart 1), $134.5^{\circ}-209.5^{\circ}$ (heart 2), $149.6^{\circ}-181.6^{\circ}$ (heart 3) and $129.7^{\circ}-207.2^{\circ}$ (heart 4) at a stretch of $5 \%$, where the first mentioned value represents the fiber orientation closest to the epicardium and the last mentioned value closest to the endocardium. During stretching, the transmural variation in fiber orientation decreased for hearts 2, 3 and 4, as shown in Table I, and the fibers approached the stretching direction, i.e. $180^{\circ}$, for all hearts. The maximal MSE-value of the ellipse fitting procedure for deriving
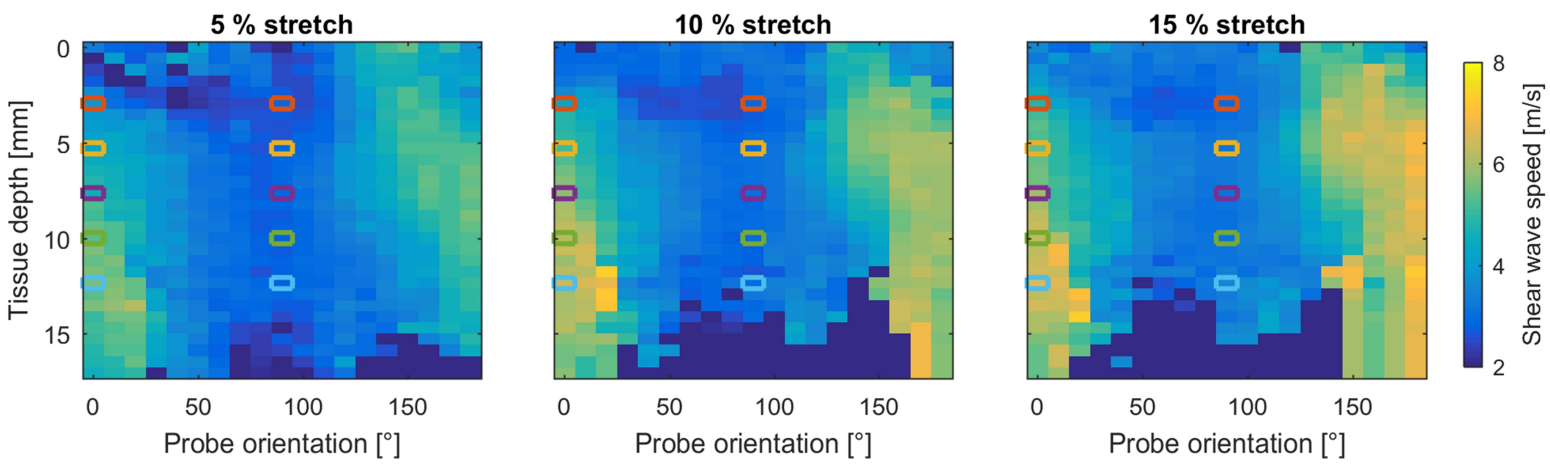

Fig. 3. Spatial anisotropy in group shear wave speed during stretching for heart $\mathrm{n}^{\circ} 3$ (raw data). Colored boxes indicate extracted locations for Figs. 5 and 6 . 

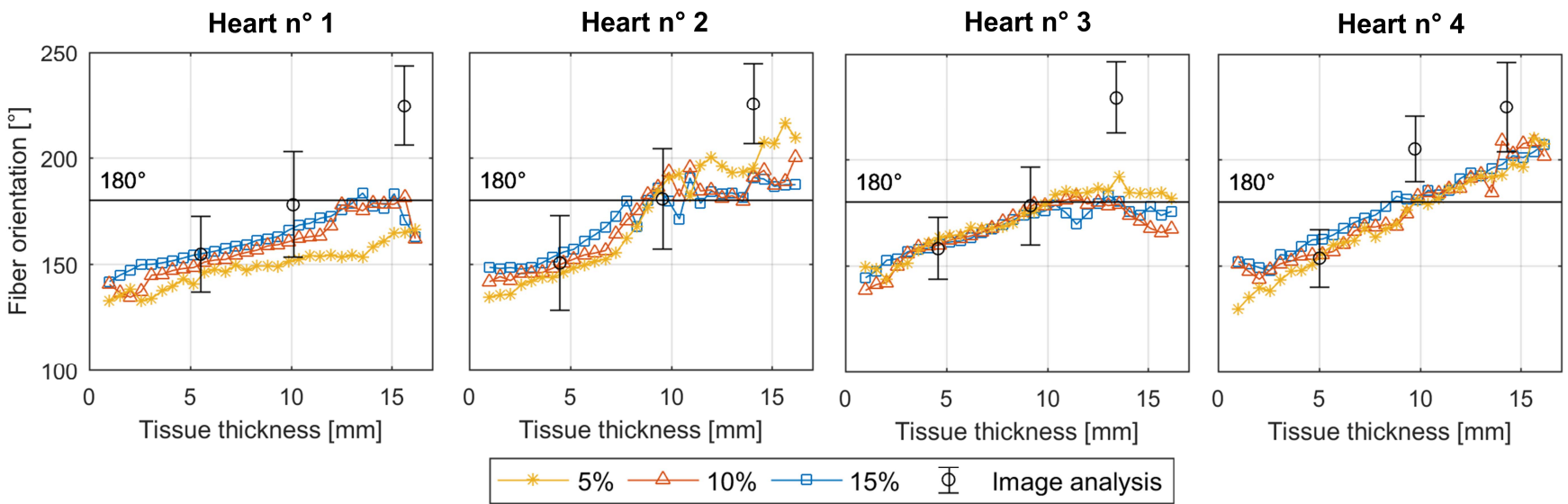

Fig. 4. Comparison of fiber orientation derived from group speed and image analysis for all hearts. Error bar represents mean with $95 \%$ confidence interval.

myocardial fiber orientation varied between 0.057 and 0.215 $(\mathrm{m} / \mathrm{s})^{2}$ for all SWE-acquisitions. The SWE-derived fiber orientation corresponded reasonably well with those of the image analysis (open symbols in black) for the epi- and midcardiac point. Data obtained at the endocardial side differed a bit more from the SWE-based values. Note that the image analysis was performed at $0 \%$ stretch.

TABLE I. TRANSMURAL FIBER VARIATION (=MAX-MIN) $\left[{ }^{\circ}\right]$.

\begin{tabular}{|c|c|c|c|}
\hline $\begin{array}{c}\text { Heart } \\
\mathbf{n}^{\circ}\end{array}$ & \multicolumn{3}{|c|}{ Stretch } \\
\cline { 2 - 4 } & $\mathbf{5 \%}$ & $\mathbf{1 0 \%}$ & $\mathbf{1 5 \%}$ \\
\hline 1 & 33.6 & 47.1 & 41.6 \\
\hline 2 & 81.8 & 58.5 & 43.1 \\
\hline 3 & 48.4 & 44.3 & 38.6 \\
\hline 4 & 80.6 & 65.2 & 59.4 \\
\hline
\end{tabular}

\section{Phase speed analysis}

Phase velocity data of the dominant excited mode (i.e. phase velocity corresponding to frequency with maximal Fourier energy) in all acquisitions are grouped in boxplots in the left panel of Fig. 5. Median values varied between 3.5$4.0 \mathrm{~m} / \mathrm{s}$ (heart 1), $2.8-3.0 \mathrm{~m} / \mathrm{s}$ (heart 2), 3.2-3.5 m/s (heart 3) and 3.1-3.4 m/s (heart 4). The $75^{\text {th }}$ percentile increased for all heart samples when stretch increases. The GLM analysis indicated that all factors heart, depth, angle, stretch, depth*angle and angle*stretch were statistically significant (mentioned in order of importance). The estimated marginal mean depicted a similar trend as for the group speeds: an increase of the maximally excited phase speed when stretch increases for probe angles $0-50^{\circ}$ and $140-180^{\circ}$, in combination with a small spatial shift of the maximum phase speed in function of probe angle.

The combined effect of stretching and shear wave anisotropy on the dispersion curve of the main excited mode is demonstrated in Fig. 6 for heart sample 3. The main excited frequencies varied from $153.5 \mathrm{~Hz}$ to $476.0 \mathrm{~Hz}$ for $0^{\circ}$ probe orientation and from $168.9 \mathrm{~Hz}$ to $307.1 \mathrm{~Hz}$ for $90^{\circ}$ probe orientation (all in the ascending part of the dispersion curve, suggesting the occurrence of dispersion) and the depths selected in this figure are indicated in Fig. 3. For the $0^{\circ}$-acquisitions, one observes an increase in the plateau-value of the dispersion curve while stretching, whereas this is less noticeable for the $90^{\circ}$-acquisitions. Furthermore, for a given stretch state, dispersion curves vary depending on the axial depth but there is no clear trend in this depth-dependency.

\section{Group vs. phase speed analysis}

The resulting group and phase speed surface at 200 and $400 \mathrm{~Hz}$ are compared across depth for heart 3 at a stretch state of 5\% in Fig. 7. At a frequency of $200 \mathrm{~Hz}$, the phase
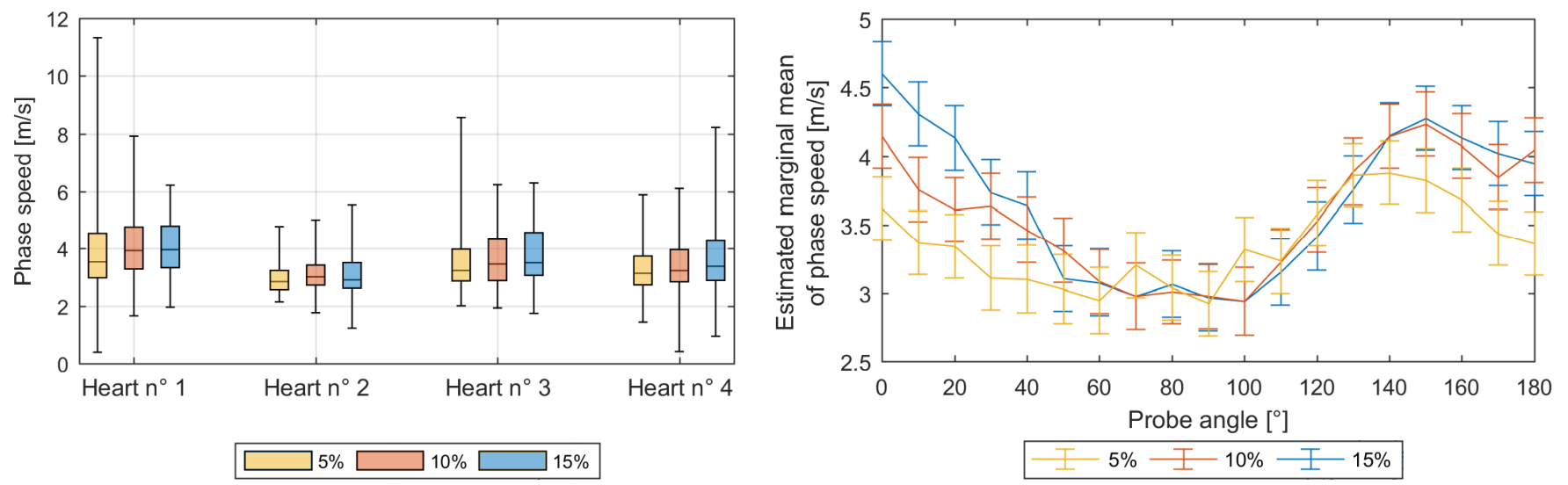

Fig. 5. Left panel - Effect of stretching on phase speed at maximum excited frequency (= frequency with highest Fourier energy) for all acquisitions with probe rotation from $0-180^{\circ}$, demonstrated in a boxplot (box represents the 3 quartiles, whiskers indicate minimum/maximum). Right panel - Combined effect of stretching and probe orientation on estimated marginal mean of phase speed values for all hearts (errorbars indicate $95 \%$ confidence interval). 

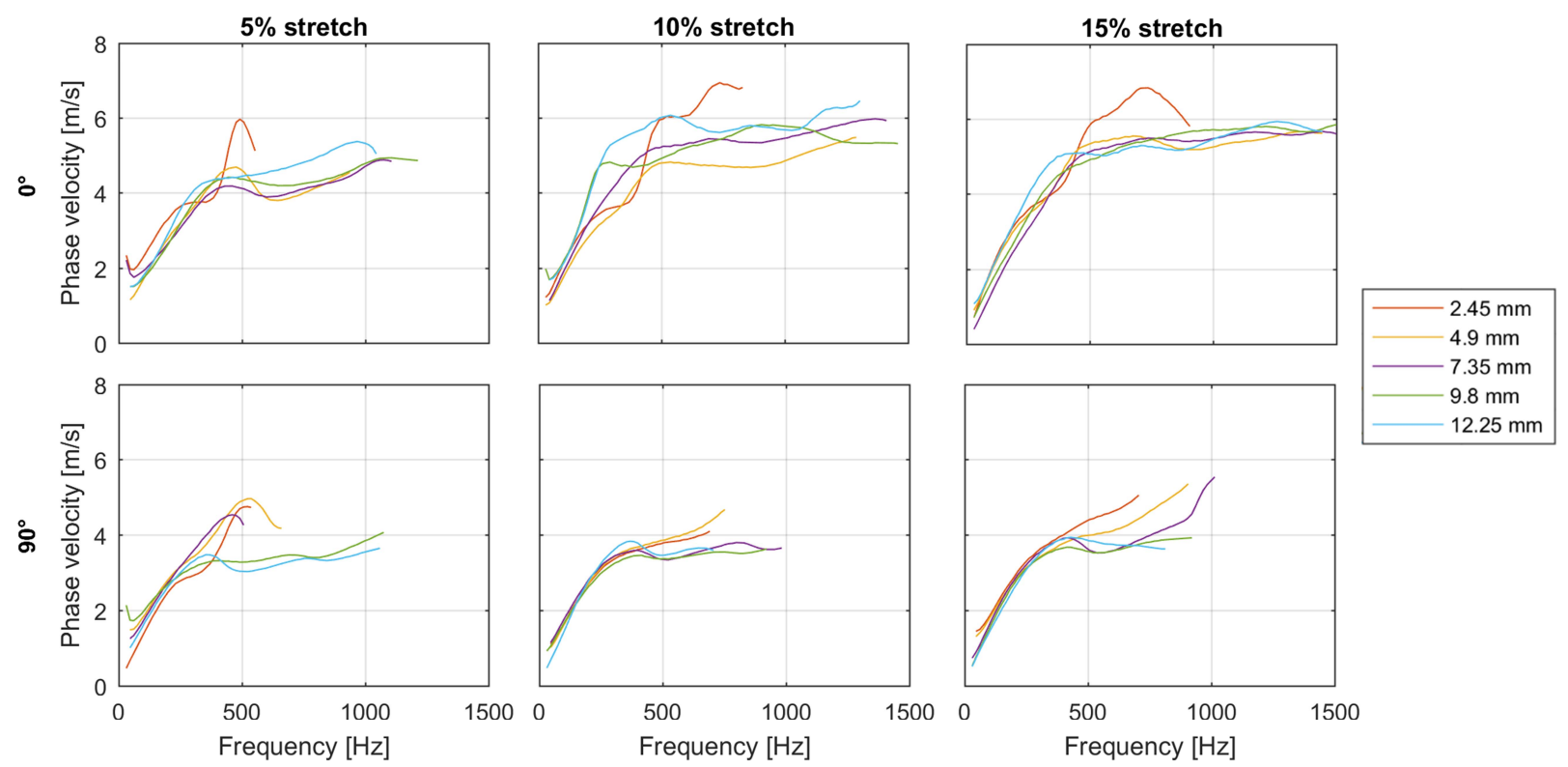

Fig. 6. Effect of stretching on dispersion curves of left shear wave for $0^{\circ}$-and $90^{\circ}$-acquisitions for 5 different depths, as indicated in Fig. 2.

speed surface is almost circular across depth, whereas the group speed surface is ellipsoidally shaped. Furthermore, the phase and group velocity values correspond around the minor axis of the group speed ellipse for all considered depths, whereas the largest difference in phase and group velocity values is obtained for probe angles along the major axis of the group speed ellipse. At a frequency of $400 \mathrm{~Hz}$, the phase speed surface becomes also ellipsoidally shaped like the group speed surface for all depths. Their velocity values are very similar for all probe angles at this frequency.

\section{DISCUSSION}

\section{A. Effect of stretching on shear wave characteristics}

As expected, group and phase speed values for a certain heart sample at a given stretch state were depth- and probe orientation-dependent, due to the varying myocardial fiber orientation across tissue thickness. The range of the resulting group speed values of all hearts corresponded to values previously reported in literature: $2-6 \mathrm{~m} / \mathrm{s}$ for in vitro porcine hearts at $0 \%$ stretch [6]. Uniaxial stretching the myocardial slab increased the group and maximally excited phase speed, as could be seen from the $75^{\text {th }}$ percentile increase, but also from the increasing predicted marginal mean of each variable during stretching. This increase was mainly caused by an increase in wave speed for probe orientation angles oriented along the fiber direction for both cases. This trend was more convincing for group speed than for phase speed, as the GLM statistical model fit showed an adjusted R-squared value of 0.804 for group speed vs. a value of 0.415 for maximally excited phase speed (also related to the fewer data points for phase speed analysis). Furthermore, for maximally excited phase speed, tissue depth was the second most relevant factor in determining marginal mean phase speed for a mean heart at a mean depth, whereas this was the fourth relevant factor for the group speed. This is also noticed from the larger 95\% confidence interval in Fig. 5 vs. Fig. 2.
Phase velocities at 200 and $400 \mathrm{~Hz}$ were also analyzed (data not shown). Marginal means of the GLM analysis showed similar trends as for the maximally excited phase velocity but models reached a lower $R$-squared value $(0.366$ and 0.252 for 200 and $400 \mathrm{~Hz}$ respectively). Nevertheless, the spatial variance in the phase velocity polar plots of Fig. 7 showed that frequency mainly affected phase speed values at probe orientations close to the fiber direction. Furthermore, phase and group speed values corresponded for cross-fiber probe orientations at the two investigated frequencies.

It should be noted that the presence of trabeculae caused considerable variation in myocardial thickness, however we analyzed only the tissue depths in which shear waves are generated by SWE. Additionally, stretching altered the myocardial thickness slightly.

\section{B. Effect of stretching on SWE-derived fiber orientation}

The extracted fiber orientation from SWE altered slightly during stretching, as the fibers became more aligned to the stretching direction of $180^{\circ}$. SWE-derived fiber orientation of heart 1 was different than that of the other hearts, probably due to difference in imaged location or intraspecific variability in fiber architecture. Fiber organization was assessed by using an ellipse based extraction method, which was more robust than utilizing maximal group velocity as surrogate for fiber angle, as done in [6]. This is also noticeable when comparing the probe orientations with maximal velocity in the group speed maps of Fig. 3 to the extracted fiber orientation in Fig. 4 for every depth.

These SWE-derived fiber orientations corresponded well with image analysis for the first two data points, but not for the last one (closest to the endocardium). Possible causes for this discrepancy are: (i) fiber direction might locally vary as this data point of the image analysis is closely located to the trabeculae in which fibers are longitudinally oriented [13], (ii) difference in location studied with both measurements 


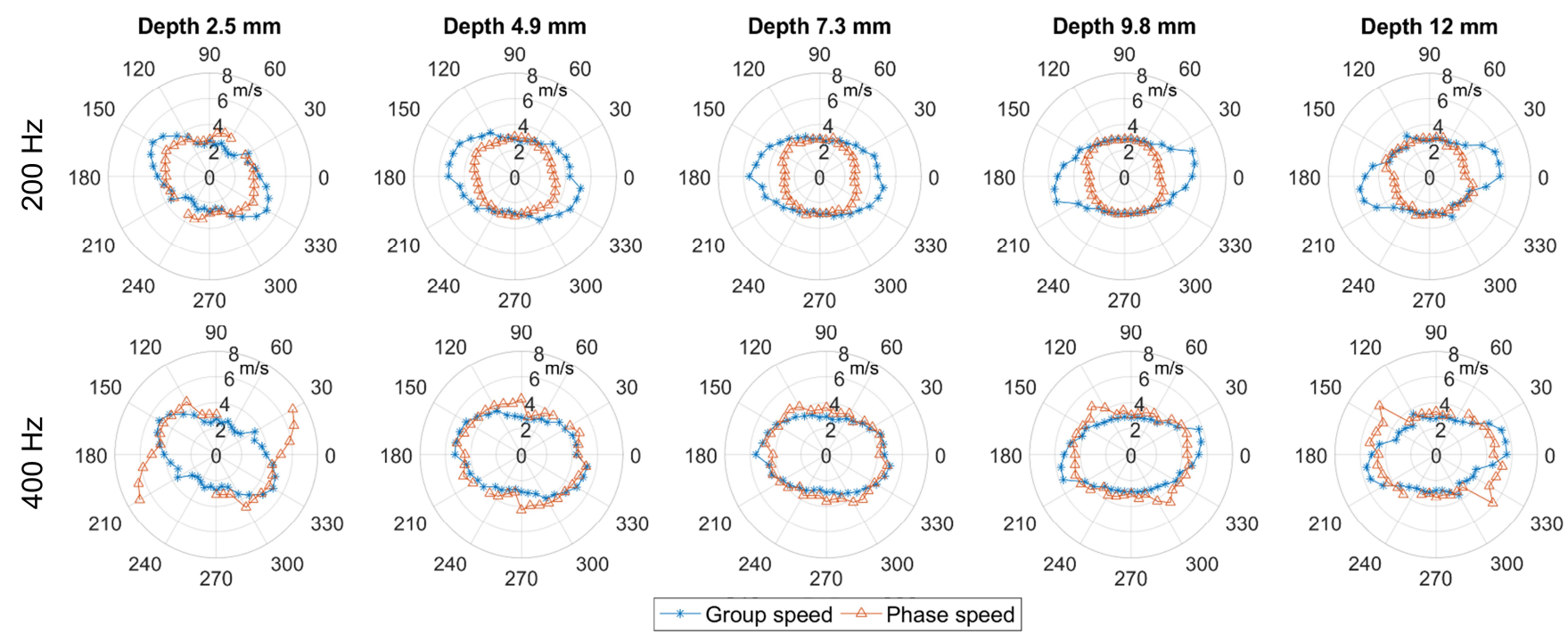

Fig. 7. Comparison of group and phase speeds at frequencies of 200 and $400 \mathrm{~Hz}$ for 5 different tissue depths in heart 3 at a stretch of $5 \%$. R-axis depicts wave speed in $[\mathrm{m} / \mathrm{s}]$, whereas $\theta$-axis represents probe orientation in $\left[{ }^{\circ}\right]$. Both group and phase speed results are averaged results of left and right shear wave.

and (iii) limited accuracy in image analysis due to suboptimal light incidence and photo resolution. Studying more than 3 samples or using a microscopic image analysis might prevent these inaccuracies.

\section{Study limitations and future work}

We considered the myocardium as transversely isotropic layers parallel to the lateral-elevational plane of the ultrasound transducer, which imposes the analyzed shear wave propagation paths in these layers, and thus does not allow a full 3D study of the 3D shear wave. It is known that the myocardial fiber architecture also has a transverse angle component next to the helix angle component, which we did not consider. This transverse angle variation is smaller than that of the helix component $\left(+9^{\circ}\right.$ to $+34^{\circ}$ vs. $-41^{\circ}$ and $\left.+66^{\circ}\right)$ [14], but future work should include more advanced analyses taking 3D shear wave tracking into account. Also, as we did not account for cardiac physiological loading, our findings can't assess the in vivo performance of cardiac SWE. A third limitation is the reliability of the extracted dispersion curves, which is undetermined for every considered location as it is dependent on the acquisition's frame rate, but also on the quality of the SWE-acquisition.

In future work, finite element modeling will complement these experiments, in which shear wave propagation is modeled by applying a temporal and spatial varying body force, representing the acoustic radiation [4]. These simulations provide access to the ground truth fiber orientation at the exact same location where shear wave analysis will be performed. This modeling should therefore resolve most of the above-mentioned study limitations.

\section{REFERENCES}

[1] P. Song et al., "Improved shear wave motion detection using pulseinversion harmonic imaging with phased array transducer," IEEE Trans Med Imaging, vol. 32, no. 12, pp. 2299-2310, 2015.
[2] M. Couade et al., "In vivo quantitative mapping of myocardial stiffening and transmural anisotropy during the cardiac cycle," IEEE Trans Med Imaging, vol. 30, no. 2, pp. 295-305, Feb 2011.

[3] M. Pernot, M. Couade, P. Mateo, B. Crozatier, R. Fischmeister, and M. Tanter, "Real-time assessment of myocardial contractility using shear wave imaging," J Am Coll Cardiol, vol. 58, no. 1, pp. 65-72, Jun 282011

[4] A. Caenen et al., "Investigating Shear Wave Physics in a Generic Pediatric Left Ventricular Model via In Vitro experiments and Finite Element Simulations," IEEE Trans Ultrason Ferroelectr Freq Control, vol. 64, no. 2, pp. 349-361, 2017.

[5] G. A. Holzapfel and R. W. Ogden, "Constitutive modelling of passive myocardium: a structurally based framework for material characterization," Philos Trans A Math Phys Eng Sci, vol. 367, no. 1902, pp. 3445-75, Sep 132009.

[6] W. N. Lee et al., "Mapping myocardial fiber orientation using echocardiography-based shear wave imaging," IEEE Trans Med Imaging, vol. 31, no. 3, pp. 554-62, Mar 2012.

[7] C. Kasai, K. Namekawa, A. Koyano, and R. Omoto, "Real-Time Two-Dimensional Blood Flow Imaging Using an Autocorrelation Technique," IEEE Trans Sonics Ultrasonics, vol. SU-32, no. 3, pp. 458-464, 1985.

[8] M. L. Palmeri, M. H. Wang, J. J. Dahl, K. D. Frinkley, and K. R. Nightingale, "Quantifying hepatic shear modulus in vivo using acoustic radiation force," Ultrasound Med Biol, vol. 34, no. 4, pp. 546-58, Apr 2008.

[9] M. Wang, B. Byram, M. Palmeri, N. Rouze, and K. Nightingale, "Imaging transverse isotropic properties of muscle by monitoring acoustic radiation force induced shear waves using a 2-D matrix ultrasound array," IEEE Trans Med Imaging, vol. 32, no. 9, pp. 167184, Sep 2013.

[10] O. Gal, "fit_ellipse.m," ed, 2003.

[11] M. Bernal, I. Nenadic, M. W. Urban, and J. F. Greenleeaf, "Material property estimation for tubes and arteries using ultrasound radiation force and analysis of propagating modes," J Acoust Soc Am, vol. 129, no. 3, pp. 1344-1354, 2011.

[12] G. Sommer et al., "Quantification of Shear Deformations and Corresponding Stresses in the Biaxially Tested Human Myocardium," Ann Biomed Eng, vol. 43, no. 10, pp. 2334-48, Oct 2015.

[13] R. A. Greenbaum, Y. H. Siew, D. G. Gibson, A. E. Becker, and R. H. Anderson, "Left ventricular fiber architecture in man," Br Heart J, vol. 45, pp. 248-263, 1981.

[14] H. Lombaert et al., "Human atlas of the cardiac fiber architecture: study on a healthy population," IEEE Trans Med Imaging, vol. 31, no. 7, pp. 1436-47, Jul 2012. 